\title{
Microstructure and Dynamics of Magnetic Iron Nanoparticles Aggregates in Protein Gels
}

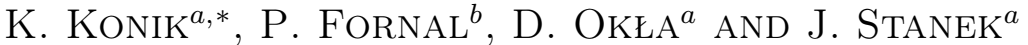 \\ ${ }^{a}$ Marian Smoluchowski Institute of Physics, Jagiellonian University, W.S. Reymonta 4, 30-059 Kraków, Poland \\ ${ }^{b}$ Institute of Physics, Cracow University of Technology, Podchorążych 1, 30-084 Kraków, Poland \\ The $60 \mathrm{~nm}$ metallic iron nanoparticles and its aggregates in gelatine gel were studied by means of optical \\ microscopy, X-ray microtomography, atomic force scanning microscopy and the Mössbauer spectroscopy. The \\ isolated nanoparticles in gel exhibit fast bounded diffusion described by Brownian movement. In the external \\ magnetic field these magnetic particles form spectacular linear aggregates whose local dynamical and magnetic \\ properties were determined from the Mössbauer spectra.
}

PACS: 75.75.Jn, 83.60.Np, 61.25.H-, 82.70.Gg

\section{Introduction}

The iron-based nanoparticles and their aggregates are the subject of the intensive studies due to their exceptional electrical, magnetic and optical properties. These nanoparticles are a highly attractive material for the creation of a new generation of devices, as, for example, nanotransistors. The most promising field of their application is, however, life science. The review of the state of knowledge and perspective in the application of magnetic nanoparticles in this field is given in Refs. [1-3]. As an example, in pharmacology the iron oxide nanoparticles are used as smart delivery systems for therapeutic agents [4-6] or as contrast agents for NMR imaging [7, 8]. Nanoparticles, profoundly covered up with a surfactant to blockade clumping, can be easily dispersed in solution and generating a new class of materials - magnetic fluids. The magnetic fluids get strongly magnetized in the magnetic field forming impressive objects [9] which may be even animated by the slowly variable external magnetic fields.

The microscopic insight into the magnetic fluids is difficult due to the short list of the physical experimental methods suitable for the study of the nanoparticles in the bulk, not transparent, soft matter media. Consequently, the description of mesoscopic properties of such systems is in the infancy. In the case of iron bearing nanoparticles the Mössbauer spectroscopy may play an important, not fully recognized yet role.

The dynamic properties of individual magnetic nanoparticles, especially in organic solutions are interesting; they may move and organize themselves in the mag-

\footnotetext{
* corresponding author; e-mail: katarzyna.konik@uj.edu.pl
}

netic field. When appropriately strong fields are used which may orient the magnetic dipoles of the particles and even link them in chains, the magneto-optical parameters of the colloids are modified [10-12].

The mobility of nonmagnetic, $\mathrm{Fe}_{2} \mathrm{O}_{3}$, nanoparticles in various media has been studied by us previously [13-15]. In this paper the formation of the aggregates of about $60 \mathrm{~nm}$ magnetic metallic Fe nanoparticles in gelatine is described. Gelatine is a processed version of collagen, a big protein, being the one of the most abundant substance in the human body. The work is based on the idea of the building in the liquid water solution of protein the aggregates of magnetic nanoparticles in form of three-dimensional assemble of needles or threads and then fixing them by the sol-gel transition in magnetic field of 1.1 $\mathrm{T}$ for the further studies.

\section{Samples}

Two types of the commercially available nanoparticles of metallic iron were chosen for the study. The first one was NanoFer 25 (producer NANO IRON) [16], the stable water solution of the $20-100 \mathrm{~nm}$ metallic iron particles with an unknown organic surfactant. Because of the broad distribution of the particles sizes, the significant amount of oxides, unknown composition and problems with the stability of the obtained gels, this very interesting material has been used only at the first stage of the investigation. The final material studied was the nanopowder of spherical metallic iron particles of the $99 \%$ purity, nominally $60-80 \mathrm{~nm}$ in diameter, supplied by American Elements [17].

The effective colloids' preparation method for nanopowder in gelatine has been worked out after several attempts. Oleic acid was chosen as the surfactant in an 
amount of about $1 \mathrm{mg}$ for $50 \mathrm{mg}$ nanoparticles, dissolved in ethanol. After evaporation of the alcohol the nanoparticles became covered with protecting layer of surfactant, about $2 \mathrm{~nm}$ thick. Then, the powder was added into the gelatine dissolved in hot water and formed, after few hours a "magnetic gel". Used gelatine was a commercially offered food product. For the microtomography and the optical studies the concentration of iron in gel was $1 \mathrm{wt} \%$, while for the Mössbauer spectroscopy the concentration was higher, typically 2 to $6 \mathrm{wt} \%$. The prepared liquids were left for few minutes to allow the sedimentation of the incidental macroscopic aggregates and afterwards placed into suitable containers. Next, the sample "frizzed" without magnetic field or in field of $1.1 \mathrm{~T}$, supplied by permanent magnet, oriented vertically or horizontally to the samples.

\section{Experimental}

\subsection{Spin-casting method and AFM microscopy}

Using the spin-casting method [18, 19], the samples of thin layers of the iron bearing gelatine were prepared. This method makes possible a rapid evaporation of solvent from a varied mixture which is placed on a backing set in very fast revolving motion. A drop of the solution was spilt on a refined silicon surface of about $1 \mathrm{~cm}^{2}$ and next distributed at speed of $\omega=2000$ rotation/s and at time $t=30 \mathrm{~s}$. The concentration of iron nanoparticles was in this case $4 \mathrm{wt} \%$ to obtain the final cover of the surface by nanoparticles in the range of few percent.

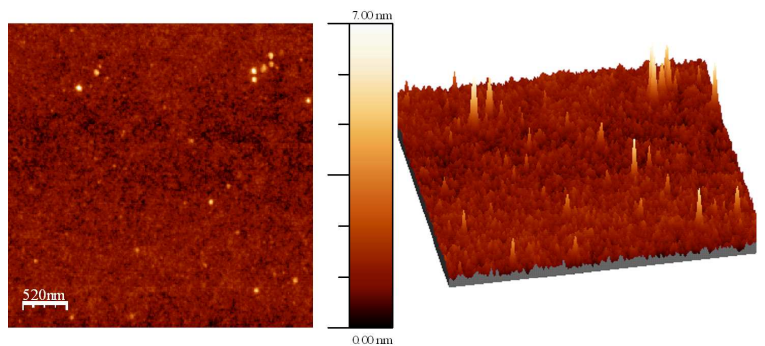

Fig. 1. Example of $2 \mathrm{D}$ and $3 \mathrm{D}$ reconstruction of the AFM scans of gelatine film with immersed iron nanoparticles.

After drying off the samples, the measurement of topography of the obtained water free gelatine film with immersed nanoparticles was made by means of atomic force microscope (AFM) with $10 \mathrm{~nm}$ resolution. An example of the 2D and 3D presentation of the obtained scans are shown in Fig. 1. The diameters of 156 particles were determined using the WSxM 5.0 Develope 3.2. The nanoparticles size distribution is presented in Fig. 2.

\subsection{Optical microscopy}

The aggregates of magnetic nanoparticles in gel were observed with the use of fluorescence microscope Optika N-400 FL, used as an optical microscope in visible light

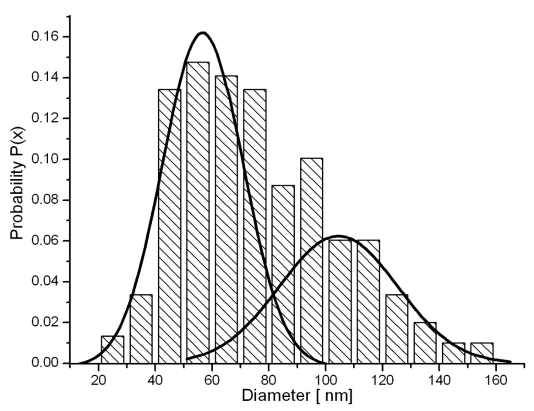

Fig. 2. Size distribution of iron nanoparticles in gelatine gel.

integrated with software Optika ${ }^{\mathrm{TM}}$ Vision Pro 3.6. The images were captured by normal light-sensitive camera with $3.5 \times 10^{9}$ pixels resolution. The images grabbed from cameras are raster images. The annotation objects created with Optika ${ }^{\mathrm{TM}}$ Vision Pro are vector graphics. It allows calibrating images, analyzing and measuring the size of the objects. The optimal magnification of specimen detail without adding unnecessary artefacts was chosen. In the case of estimating the distance between the needles the magnification of 40 was selected and increased to 100 for the observation of the individual object.

\subsection{X-ray microtomography}

The 3D image of the system of aggregates in the whole sample has been obtained with the Microtomography Skyscan 1172 [20]. The maximal resolution of this tomograph is $0.4 \mu \mathrm{m}$. However, in this case the measuring time for the typical $1 \mathrm{~cm}$ sample reaches $24 \mathrm{~h}$. The temperature at the sample place increases up to $30^{\circ} \mathrm{C}$. These two factors may destroy our gelatine sample during the measurements. Thus, the lower resolution, $1.4 \mu \mathrm{m}$ was chosen at the present stage of the investigation. For this resolution measuring time was $6 \mathrm{~h}$ and the numerical reconstruction time was $5 \mathrm{~h}$. The microtomographer worked with the voltage of $50 \mathrm{kV}$ and the current $100 \mu \mathrm{A}$.

\subsection{Mössbauer spectroscopy}

The application of the Mössbauer spectroscopy to the determination of Brownian movement of microobjects bearing nuclear probe, here ${ }^{57} \mathrm{Fe}$, has been recognized soon after the discovery of the recoil-free resonant absorption of gamma rays. When each moving particle in the sample may be treated as classical absorbers, the originally Lorentzian line shape, $L(v)$, of the resonant absorption becomes convoluted with the velocity spectrum of the particles, $p(V)$, and the final line shape, $I(v)$ is

$$
I(v)=\int p(V) L(v-V) \mathrm{d} V .
$$

Here, $v$ and $V$ are the source velocity and the velocity of the particle, respectively, both vs. the laboratory frame. If $p(V)$ is Gaussian, $I(v)$ is the Voigt line. 


$$
I\left(v, \sigma_{v}, \Gamma\right)=\int \frac{\mathrm{e}^{-V^{2} /\left(2 \sigma_{v}^{2}\right)}}{\sigma_{v} \sqrt{2 \pi}} \frac{\Gamma}{\pi\left[(v-V)^{2}+\Gamma^{2}\right]} \mathrm{d} V .
$$

The $\sigma_{v}$ parameter is just the standard deviation of the velocity distribution of the particles, $\Gamma$ is the experimental line width. Here, the velocity of the particle, $V$, is $V=\Delta x / \Delta t$, where $\Delta x$ is the displacement of the particles in $\Delta t$ time, $\Delta t=141 \mathrm{~ns}$, the lifetime of the $14.4 \mathrm{keV}$ nuclear level, the characteristic observation time for the ${ }^{57} \mathrm{Fe}$ Mössbauer spectroscopy.

The Mössbauer spectra were recorded at room temperature in the transmission geometry with the use of the Wissel spectrometer [21]. For the measurements in the external field of $1.1 \mathrm{~T}$ the permanent magnet, the same as that used for preparation of the needles was applied. Typically, one spectrum was collected in 512 channels (before folding) for $24 \mathrm{~h}$. The activity of the ${ }^{57} \mathrm{Co} / \mathrm{Rh}$ source was $50 \mathrm{mCi}$.

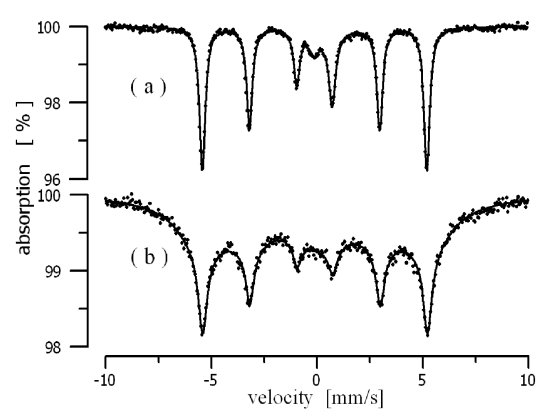

Fig. 3. The Mössbauer spectra of NanoFerm 25: (a) powder sample, (b) sample in gelatine gel.

The Mössbauer spectra of the nanoparticles from NanoFer 25 are shown in Fig. 3. Because of the arguments given in Sect. 2 this material has been excluded, for the time being, from the further study and we focused on the nanopowder from American Elements.

For the Mössbauer experiments the suspension of iron nanoparticles in gelatine were placed in a thin wall plastic container, $20 \mathrm{~mm}$ in diameter and $8 \mathrm{~mm}$ thick. For each sample the final amount of the iron, being in the range between 5 and $8 \mathrm{mg} / \mathrm{cm}^{2}$, was determined from the comparison of the total area of the Mössbauer spectrum with the area of the spectrum of the reference absorber, see Fig. 4a, of the known thickness of iron nanoparticles $\left(6.7 \mathrm{mg} / \mathrm{cm}^{2}\right)$ mixed with lucid powder and pressed in the form of pellet. Thus, the derived concentration of the particles in gel was about $5 \times 10^{12}$ particles $/ \mathrm{cm}^{3}$, or their volume fraction was about $10^{-3}$. Consequently, the average particle-particle distance is about $500 \mathrm{~nm}$. The sol-gel transition appeared at room temperature without the external magnetic field or in the homogeneous magnetic field of $1.1 \mathrm{~T}$.

The numerical analysis of the spectra of nanoparticles in gel was complicated. The Voigt line shape was assumed for the fit. It turned out that for the proper reproduction of the experimental resonant line shape the

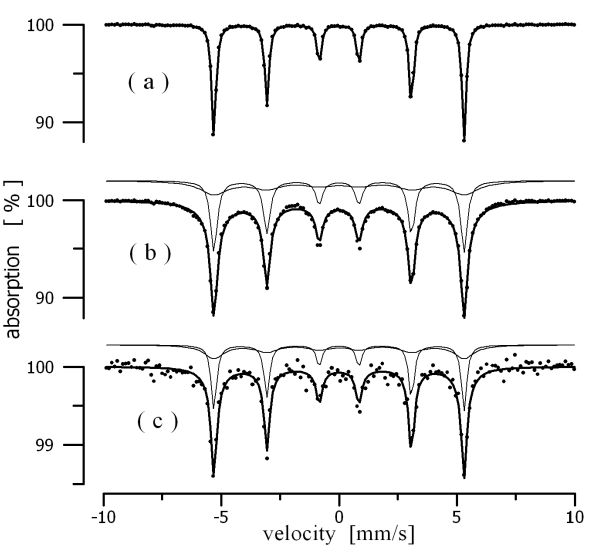

Fig. 4. The Mössbauer spectra of iron nanoparticles (American Elements): (a) sample in form of a pressed pellet, treated as the reference, (b) nanoparticles in gel formed without magnetic field, (c) needles formed in gel in magnetic field, measured in the field parallel to the needles.

superimposing of at least two Voigt lines with different widths must be assumed, see Fig. 4b,c. This means that the velocity profile is the sum of two Gaussians. Fortunately, the line widths in the sextet are obviously identical and the positions of the lines are known from the reference sample, see Fig. 4a. This significantly reduced the number of fitted parameters.

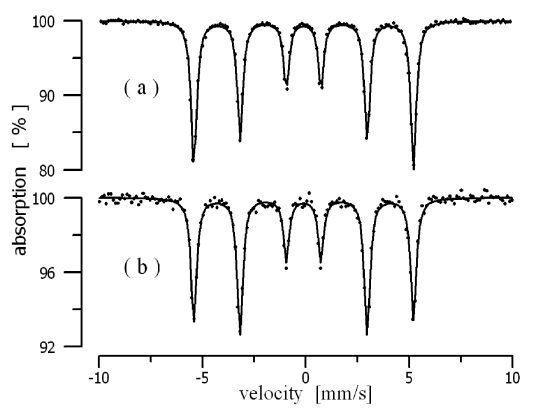

Fig. 5. The Mössbauer spectra of metallic iron foil measured without external magnetic field (a), and in external magnetic field of $1.1 \mathrm{~T}$, parallel to the foil plane (b).

Usually, when the magnitude of the hyperfine interaction parameters are studied, the samples containing few mg of iron per $\mathrm{cm}^{2}$, may be treated as thin absorbers, especially in the case of the magnetically split spectra. The finite thickness causes the broadening of the line and disturbs the line intensity ratio. In the present case these parameters are crucial: they determine the mobility of the particles and the magnetic texture of the sample, respectively. In particular, in the magnetic split Mössbauer spectra of ${ }^{57} \mathrm{Fe}$, the line intensity ratio within the Zeeman pattern is $3: x: 1: 1: x: 3$, where $x$ varies from 0 for the magnetic polarization parallel to the $\gamma$-beam, through 2 for the random distribution of the magnetic moment, up 
to 4 for the magnetization perpendicular to the $\gamma$-beam. As an example, the spectrum of the iron calibration foil measured in the $1.1 \mathrm{~T}$ magnetic field parallel to foil and perpendicular to the $\gamma$-beam is shown in Fig. 5b. The intense 2nd and 5th lines prove the strong in plane magnetic texture of the foil. Thus, to reduce the influence of the sample thickness on the line intensity ratio we performed the thickness correction of the spectra using the MOSSMOD code [22]. The presented spectra are just the corrected one for the thin absorber limit; it should be noted, however, that such correction had miner influence on the final results.

\section{Results}

\subsection{Size distribution of the nanoparticles}

The asymmetric size distribution profile, see Fig. 2 were fitted with two Gaussians with maxima at $57 \mathrm{~nm}$ and $107 \mathrm{~nm}$ and standard deviation of $14 \mathrm{~nm}$ and $21 \mathrm{~nm}$, respectively. The first peak corresponds to the isolated particles. Its position is slightly below the $60 \mathrm{~nm}$ to $80 \mathrm{~nm}$ range given by the producer. Because the nanoparticles are covered with gelatine film of about $20 \mathrm{~nm}$ thickness, a systematic error in the determination of the particle diameter cannot be excluded. The second peak should be assigned to the dimeric state of the nanoparticles. The relative fractions of these two forms of the nanoparticles are 64 and $36 \%$, respectively.

\subsection{Morphology of aggregates}

The originally black or grey colloid of iron nanoparticles becomes transparent in the magnetic field due to the formation of the assembly of the visible needles, see Fig. 6. The shapes of individual needles, observed with optical microscope are quite impressive, see Fig. 7. The "screw-like" objects were formed after partial drying of the sample causing its contraction.

The view of gelatine surface with needles directed perpendicular to the plane of the photograph is showed in Fig. 8a serving the data for the determination of the needle diameter distribution. Here, the public domain Java image processing program - ImageJ for Microscopy from Macbiophotonics has been applied. ImageJ is a free, very versatile program package for image processing and manipulation. One of its options is the automatic determination of needles diameter distributions in the following steps: (a) to calibrate the image magnification, (b) to find threshold for the image, see Fig. 8b, (c) to analyze particles, see Fig. 8c. There are some problems to determine the diameter of all needles unambiguously, for example the particles at the edge of thick areas are not clearly recognizable and the particle close together might not be resolved. The final distribution of the needles' diameter is presented in Fig. 9. It turns out that in spite of the fact that the thin needles, less than $10 \mu \mathrm{m}$ in diameter are the most frequent, the typical diameter of the needles varies between 20 and $100 \mu \mathrm{m}$.

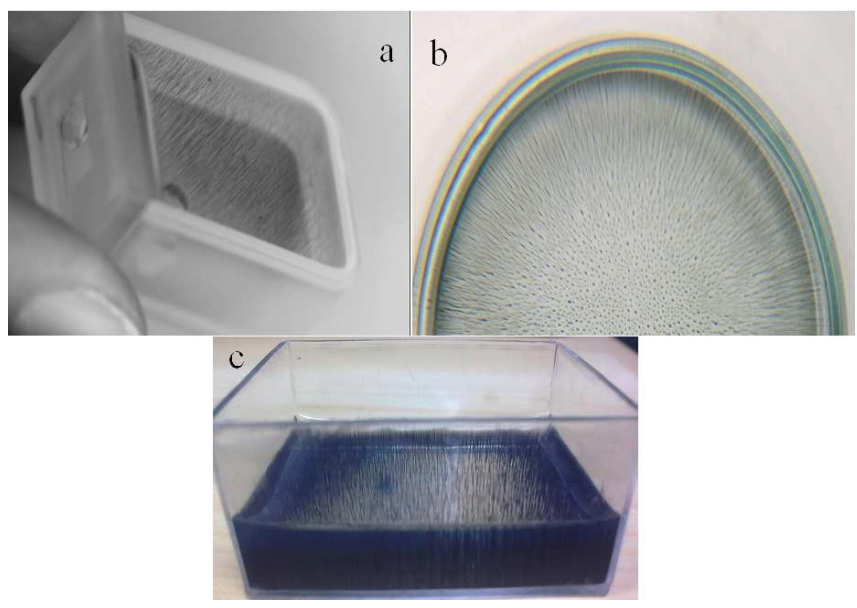

Fig. 6. The assembly of metallic needles formed from iron nanoparticles in the vertical magnetic field of $1.1 \mathrm{~T}$ and stabilized in the gelatine gel (a). The tops of hills, forming a regular structure of radially arranged clusters of magnetic particles (b). Gelatine surface, when the liquid was removed from the magnetic field with formed needles (c).

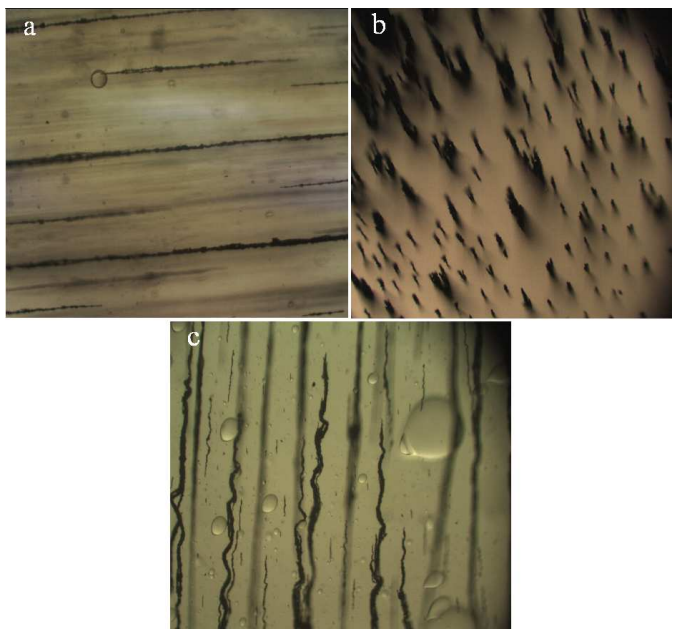

Fig. 7. The aggregates of iron nanoparticles in gelatine gel observed with optical microscope with the magnification 100. The "screw-like" objects were formed after partial drying of the sample causing its contraction (c).

The visual inspection of $2 \mathrm{D}$ distribution of the needles oriented perpendicularly to sample surface suggests some small degree of their ordering. In Fig. 10 the distribution of needles distances, is presented. It shows that there is a minimal needle-needle distance of about $100 \mu \mathrm{m}$. The possible two-dimensional ordering of these object, which would lead to some maxima in the radial distribution function, is questionable.

The three-dimensional reconstruction of the system of aggregates is shown in Fig. 11. It turns out that the needles consist of big, approximately spherical aggregates of nanoparticles. However, the poor resolution of the 


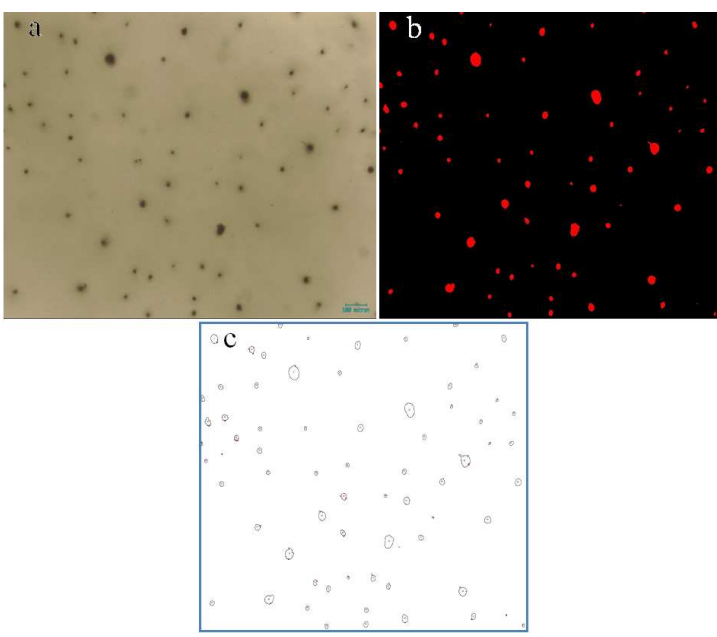

Fig. 8. The view of gelatine surface with needles directed perpendicular to the plane of the photograph (a), the threshold for the particles in the image (b), the outlined analyzed particles (c).

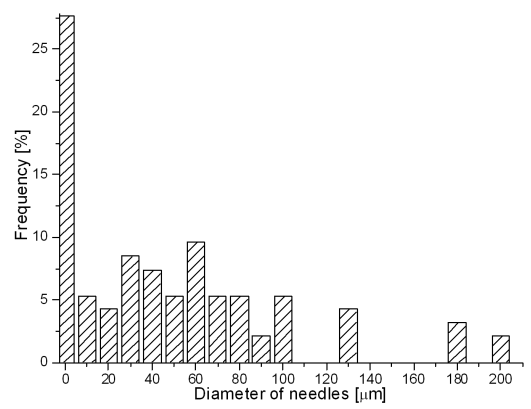

Fig. 9. The distribution of needles' diameter.

microtomograph does not allow the detailed analysis of the mesoscopic structure of the needles.

\subsection{Magnetic and dynamical properties}

The good quality Mössbauer spectrum of the reference iron nanoparticles pressed in the pellet is showed in Fig. 5a. It is identical to the calibration spectra of

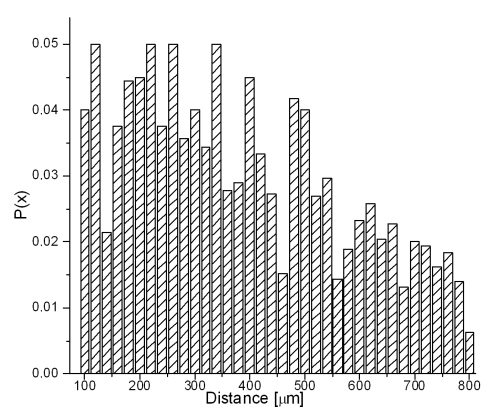

Fig. 10. The radial distance distribution of needles in the plane perpendicular to the applied magnetic field.

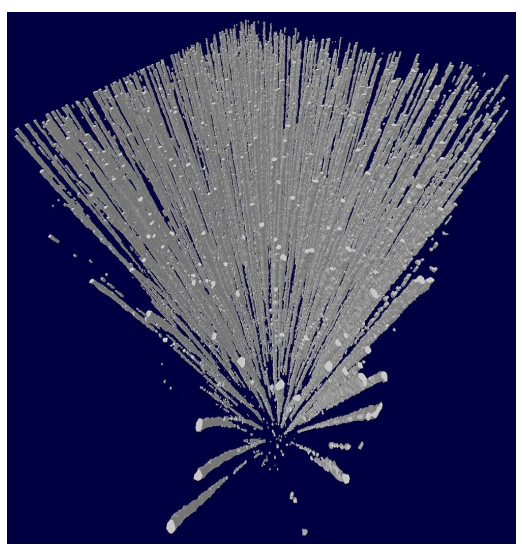

Fig. 11. The 3D reconstruction of microtomography of the needles formed from iron nanoparticles in gelatine gel in external magnetic field of $1.1 \mathrm{~T}$.

bulk Armco iron. Surprisingly, in spite of the big surface fraction in the nanopowder (about $700 \mathrm{~cm}^{2}$ per $1 \mathrm{~cm}^{2}$ of the sample absorber) no sign of any oxidation is seen. The absorption lines are quite narrow, the half widths at half intensity is $\Gamma=0.106 \mathrm{~mm} / \mathrm{s}$ which shows that there is no distribution of the hyperfine magnetic field over the particles. The 3:2:1 intensity ratio excludes the magnetic texture of the pellet.

The spectrum of the nanoparticles in gelatine gel formed and recorded at ambient conditions was shown already in Fig. 5b. The spectrum of this sample recorded in the magnetic field is identical. As in the case of the pellet, there is no magnetic texture of the sample, even in the external magnetic field. The broadening of the resonance lines, identical for each line, is the evidence of the Brownian movement of the nanoparticles. The two fractions in the spectrum point out two different types of nanoparticles, distinguished by their mobility. The mass fractions, proportional to corresponding subspectra areas, of the more mobile particles with $\sigma_{v}=2.24 \mathrm{~mm} / \mathrm{s}$ and the less mobile with $\sigma_{v}=0.16 \mathrm{~mm} / \mathrm{s}$ are $40 \%$ and $60 \%$, respectively.

An example of the spectra of needles formed in magnetic field is presented in Fig. 5c. In all cases the $\gamma$ -beam was perpendicular to the needles. The aggregation of the nanoparticles reduces their mobility. Now, the $\sigma_{v}$ of the more and less mobile particles is $1.63 \mathrm{~mm} / \mathrm{s}$ and 0.09 , respectively, with the mass fractions of 0.3 and 0.7 , respectively. The orientation magnetic moments of iron atoms without magnetic field is random. The magnetic field of $1.1 \mathrm{~T}$ aligns the domains only partly. In the case of field parallel to the needles the line intensity ratio in the Mössbauer spectrum, shown in Fig. $5 \mathrm{c}$ is 3:2.25:1:1:2.25:3 which means that $15 \%$ of the particles is preferentially oriented along the needles, while for the field perpendicular to the needles this ratio was 3:2.1:1:2.1:3 which means that the preferential orientation of the magnetic moments, if any, is in fact in the error limit. 


\section{Discussion}

The broad distribution of the needles' diameter and their poorly ordered spatial structure as well as the quasi fractal architecture points out that the process of the aggregation of the magnetic nanoparticles in magnetic colloids is quite complicated. The shape of the final objects probably depends on the local fluctuation of the concentration of nanoparticles, inhomogeneity of the magnetic field, temperature and viscosity of the solvent. On the other hand, it shows that there are perspectives to develop a new technique of the nanotechnology leading for the new microdevices. This aim may be achieved after determination of the microscopic, dynamical and magnetic properties of the nanoparticles in the gels.

The broadening of the resonant absorption line of the Mössbauer spectrum, expressed in $\mathrm{mm} / \mathrm{s}$ is the standard deviation of the Gaussian distribution of the one-dimensional (in the direction of the gamma beam) velocity of the particles. It is amazing that this experimental method may measure the distribution of the particles velocities, averaged in the 141 ns observation time, in the range of $0.1 \mathrm{~mm} / \mathrm{s}$ which means that the nanoparticles displacement of order $10^{-11} \mathrm{~nm}$, i.e. $\approx 0.1 \%$ of their diameters, is detectable.

The Einstein-Smoluchowski theory of the Brownian movement gives the expression for the mean square displacement, $\sigma_{x}^{2}$ of the particles with radius $r$ in the medium with the viscosity $\eta$ :

$$
\sigma_{x}^{2}=\frac{2 k T \Delta t}{6 \pi \eta r}
$$

For $60 \mathrm{~nm}$ particles $(r=30 \mathrm{~nm})$ in water $(\eta=$ $0.001 \mathrm{~Pa} \mathrm{~s}), T=300 \mathrm{~K}$ and $\Delta t=141 \mathrm{~ns}, \sigma_{x}=1.4 \mathrm{~nm}$, which leads to the $\sigma_{v}=10 \mathrm{~mm} / \mathrm{s}$. In gelatine gel, of the macroscopic viscosity $10^{5}$ times higher than this of water, $\sigma_{v} \approx 0 \mathrm{~mm} / \mathrm{s}$. In the studied case $\sigma_{v}$ of the isolated particles is in the range of $1 \mathrm{~mm} / \mathrm{s}$, thus the viscosity must be treated locally. The problem of scaling form of viscosity and the diffusion has been studied intensively [23-27]. The iron nanoparticles in the gelatine gel exhibit the bounded diffusion within the water voids, about $1 \mu \mathrm{m}$ in diameter, kept by the three-dimensional network of the amine chains.

There are three possible explanations of the reduction of the mobility:

(i) a microfriction with amine chains,

(ii) instead of particle radius, the hydrodynamical radius, which includes a layer of solvent molecules bonded to the nanoparticles surface should be considered. The thickness of such layer may be in the range of $100 \mathrm{~nm}$ [13]. The concept of hydrodynamical radius explains the surprising fact that in the needles the mobility of nanoparticles is only reduced by about $50 \%$ and is not fully eliminated as in pellet. It may be postulated that in these aggregates the nanoparticles are not directly bounded but only attached to each other being separated by water or collagen layer. (iii) The magnetic damping of the Brownian movement. Such effect has been postulated from the comparison of the mobility of the magnetic $(\mathrm{Fe})$ and not magnetic $\left(\mathrm{Fe}_{2} \mathrm{O}_{3}\right)$ nanoparticles in sediments [23].

The appearance of the two types of nanoparticles, distinguished by their velocity, is explained by their size distribution which shows that about $36 \%$ of observed particles are dimers of nanoparticles. Thus, 64\%/(0.64+ $2 \times 0.36)=48 \%$ of nanoparticles are monomers. Accordingly, the "broad line" component in the Mössbauer spectra whose area is $40 \%$ of the total may be assigned to the mobile isolated nanoparticles and the "narrow line" component to their aggregates.

The magnetic properties of this new class of materials appear interesting. The particles with the diameter in the range of tens nanometer are frequently considered as single domains. However, the $60 \mathrm{~nm}$ iron particle contains about $10^{7}$ atoms with magnetic moment of $2.2 \mu_{\mathrm{B}}$. Thus, in the single domain case such particle should have magnetic moment $\mu=2 \times 10^{-16} \mathrm{~J} \mathrm{~T}^{-1}$. Consequently, its interaction energy, $E$, with the field, $B=1.1 \mathrm{~T}$, would be $E=\mu B=1.4 \times 10^{3} \mathrm{eV}$, which means that the system of isolated iron nanoparticles should be fully polarized. This is in the discrepancy with the Mössbauer data which shows random orientation of the magnetic moments of nanoparticles at room temperature. This means that the energy interaction $E$ is in the range of $k T=2.5 \times 10^{-2} \mathrm{eV}$.

The magnetic properties of the needles formed in magnetic field are surprising. In spite of the high morphological anisotropy the orientation of the individual magnetic nanoparticles is random with the exception when the magnetic field applied parallel to the needles causes a tiny polarisation.

\section{Conclusions}

The method of the preparation of the suspension of the isolated iron nanoparticles or its tiny aggregates in amine gel was worked out. The prepared specimens serve the opportunity to study the local dynamical properties of nanoscale objects in soft matter which may help in the better understanding of transport processes at subcellular level. The further investigation of the reciprocal interaction of the magnetic nanoparticles may help in the description of formation of their impressive aggregates in external magnetic field which may be a starting point for the new nano engineering.

\section{Acknowledgments}

We thank Ms. Joanna Zemła for her help in the determination of the nanoparticle size distribution by means of atomic force microscope and Mr. Bartosz Leszczyński for the $3 \mathrm{D}$ visualization of iron needles. The Mössbauer spectroscopy and microtomography studies were carried out with the equipment purchased thanks to the financial support of the European Regional Development Fund in the framework of the Polish Innovation Economy Operational Program (contract no. POIG.02.01.00-12-023/08). 


\section{References}

[1] A. Ślawska-Waniewska, Postępy Fizyki 55, 157 (2004) (in Polish).

[2] C.C. Berry, A.S.G. Curtis, J. Phys. D, Appl. Phys. 36, 198 (2003).

[3] S.A. Corr, A. O' Byrne, Y.K. Gun'ko, S. Ghosh, D.F. Brougham, S. Mitchell, Y. Volkov, A. Prina-Mello, Chem. Commun., 4474 (2006).

[4] A. Figuerola, R. Corato, L. Manna, T. Pellegrino, Pharmacol. Res. 18, 126 (2010).

[5] M.K. Yu, Y.Y. Jeong, J. Park, S. Park, J.W. Kim, J.J. Min, K. Kim, S. Jon, Angew. Chem. Int. Ed. 47, $5362(2008)$

[6] E. Ruoslahti, S.N. Bhatia, M.J. Sailor, J. Cell Biol. 188, 6759 (2010).

[7] G.R. Reddy, M.S. Bhojani, P. McConville, J. Moody, B.A. Moffat, D.E. Hall, G. Kim, Y.-E.L. Koo, M.J. Woolliscroft, J.V. Sugai, T.D. Johnson, M.A. Philbert, R. Kopelman, A. Rehemtulla, B.D. Ross, Clin. Cancer Res. 12, 6677 (2006).

[8] C.G. Hadjipanayis, M.J. Bonder, S. Balakrishnan, X. Wang, H. Mao, G.C. Hadjipanayis, Small 4, 1925 (2008).

[9] www.kodama.hc.eue.ac.jp/protrudeflow/ index.html .

[10] J. Bacri, V. Cabuil, R. Perzynski, R. Massart, D. Salin, J. Magn. Magn. Mater. 65, 285 (1987).

[11] L. Zhao, J. Appl. Phys. 92, 1128 (2002).

[12] D. Jamon, D. Zins, H. Royer, V. Cabuil, C. Dovier, J.J. Rousseau, Indian J. Eng. Mater. Sci. 11, 237 (2004).

[13] P. Fornal, J. Stanek, Acta Phys. Pol. A 114, 1667 (2008).
[14] P. Fornal, J. Stanek, A. Wilk, Hyperfine Interact. 185, 181 (2008).

[15] J. Stanek, P. Fornal, Hyperfine Interact. 190, 75 (2009).

[16] http://www.nanoiron.cz/en/nanofer-25 ad nanof ern25.

[17] http://www . americanelements.com/fenp.html .

[18] C.J. Lawrence, Phys. Fluids 31, 2786 (1988).

[19] S. Nilsson, A. Bernasik, A. Budkowski, E. Moons, Macromolecules 40, 8291 (2007).

[20] http://www.skyscan.be/products/1172.htm .

[21] http://www.wissel-gmbh.de/ index $\cdot$ php?option=com_content\&task=view\&id=102 \&Itemid $=138$

[22] D.F.G. Rancourt, Nucl. Instrum. Methods Phys. Res. B 44, 199 (1989).

[23] P. Fornal, J. Stanek, Acta Phys. Pol. A 119, 12 (2011).

[24] J. Gapiński, J. Szymanski, A. Wilk, J. Kohlbrecher, A. Patkowski, R. Holyst, Langmuir 26, 9304 (2010).

[25] J. Szymański, A. Patkowski, A. Wilk, P. Garstecki, R. Hołyst, Phys. Chem. Lett. B 110, 25593 (2006).

[26] R. Hołyst, A. Bielejewska, J. Szymanski, A. Wilk, A. Patkowski, J. Gapinski, A. Zywocinski, T. Kalwarczyk, E. Kalwarczyk, M. Tabaka, N. Ziebacz, S.A. Wieczorek, Phys. Chem. Chem. Phys. 11, 9025 (2009).

[27] M. Barski, Czasopismo Techniczne, Mechanika, Wydawnictwo Politechniki Krakowskiej 12, 3 (2009) (in Polish). 